\title{
Efficient solution of fluid-structure vibration problems
}

\author{
Mario Mellado and Rodolfo Rodríguez ${ }^{1}$ \\ Departamento de Ingeniería Matemática, Universidad de Concepción, \\ Casilla 160-C, Concepción, Chile.
}

\begin{abstract}
This paper deals with the numerical computation of elastoacoustic vibration modes. We consider a redundant description of the fluid by means of pressure and displacement potential variables introduced by Morand and Ohayon. We analize a finite element discretization leading to a well posed symmetric banded eigenvalue problem. An iterative algorithm requiring to solve sparse linear systems with one degree of freedom per fluid node is obtained. We show that, for acoustic modes, this method coincides with a consistent discretization of the standard potential formulation. Numerical experiments are included to validate the proposed methodology for elastoacoustic vibrations.
\end{abstract}

\section{Introduction}

During the last years increasing attention has been paid to the numerical computation of fluid-solid interactions because of their interest in many engineering problems. See, for instance, the monographs by Morand and Ohayon [9] and by Conca et al. [5], both including large lists of references.

This paper deals with the numerical solution of a typical fluid-structure interaction, the so-called interior elastoacoustic vibration problem, which concerns to determining the free vibration modes of an inviscid compressible fluid contained in a linear elastic solid. We assume that gravity effects can be neglected.

To compute the acoustic modes of a fluid alone, the fluid is typically described either by pressure or by displacement potential variables. Such formulations have the advantage of using only one degree of freedom per node in the fluid. However, when the fluid is coupled with a solid, both lead to non-symmetric

${ }^{1}$ Supported by FONDECYT (Chile) through grant No. 1.960.615. 
eigenvalue problems (see, for instance, the book by Zienkiewicz and Taylor [11]).

To avoid this drawback, different variables have been proposed to describe the fluid: Everstine [6] used the velocity potential obtaining a symmetric but quadratic eigenvalue problem; Kiefling and Feng [7] considered displacement variables, but they show that spurious modes arise when standard finite elements are used. More recently, alternative discretizations avoiding these spurious modes have been proposed by Chen and Taylor [4] and Bermúdez and Rodríguez [2]. However, all these formulations require to discretize a vector field (displacements) instead of a scalar one (either pressure or potential).

On the other hand, Morand and Ohayon [8] considered a redundant description of the fluid by using both, pressure and displacement potential. By so doing, they obtained a variational spectral problem which, when discretized by finite elements, yields a symmetric eigenvalue problem involving sparse matrices. To solve it, they propose to eliminate either the pressure or the potential degrees of freedom. However, any of these procedures destroy the sparseness of the matrices. See chapter 8 of the book by Morand and Ohayon [9] for further details and alternative approachs.

Indeed, it is not necessary to eliminate in advance neither the pressure nor the potential degrees of freedom. In fact, we show that the complete sparse symmetric eigenvalue problem obtained by Morand and Ohayon [8] is well suited for numerical computations. We consider a finite element discretization involving piecewise constant approximations of the pressure. Such procedure allows to eliminate the degrees of freedom corresponding to this variable at a further stage without destroying the sparseness of the involved matrices.

We describe how our strategy can be combined with a shifted inverse power method yielding an algorithm to compute the vibration modes of the coupled system which only requires to use sparse matrices and one degree of freedom per fluid node. Such strategy could be easily adapted to any "shift and invert" eigenvalue solver.

We also show that our discretization, when used to compute the acoustic modes of the fluid alone, coincides with a consistent discretization of the standard formulation of the problem by displacement potential variables. Finally, we present numerical results validating the proposed methodology and showing its good performance. 


\section{Statement of the problem}

Let $\Omega_{F}$ and $\Omega_{S}$ be the domains occupied by the fluid and the solid, respectively, as shown in Figure 1. The structure is fixed along a part of its boundary $\Gamma_{D}$ and free of stress on other part $\Gamma_{N} . \Gamma_{I}$ denotes the interface between the solid and the fluid. $\nu^{S}$ and $\nu^{F}$ are the normal vectors pointing outwards $\Omega_{S}$ and $\Omega_{F}$, respectively.

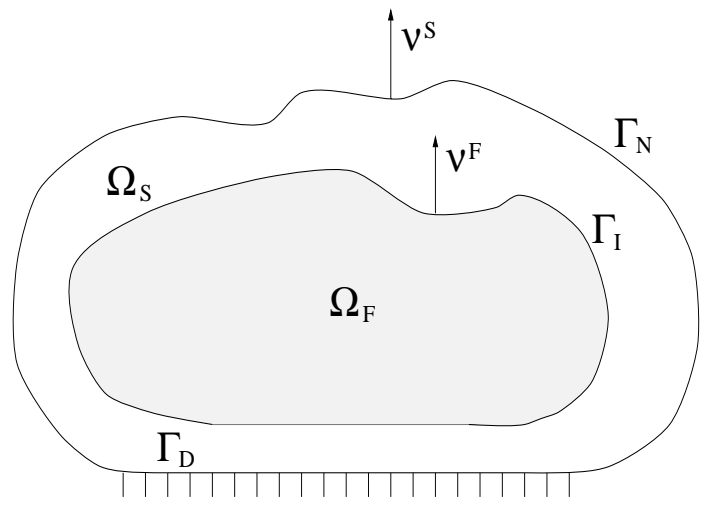

Fig. 1. Fluid-Structure System.

We consider the classical acoustic aproximation for the small amplitude motions of an ideal inviscid barotropic fluid contained in a linear elastic structure. We suppose that body forces and initial stresses at equilibrium are negligible and we ignore them. So, in absence of external forces, the basic equations are:

$$
\begin{array}{rlr}
\operatorname{div} \sigma\left(u_{S}\right)-\rho_{S} \frac{\partial^{2} u_{S}}{\partial t^{2}}=0, & \text { in } \Omega_{S}, & t>0, \\
\sigma\left(u_{S}\right) \nu^{S}=0, & \text { on } \Gamma_{N}, & t \geq 0, \\
u_{S}=0, & \text { on } \Gamma_{D}, & t \geq 0, \\
-\sigma\left(u_{S}\right) \nu^{F}=p \nu^{F}, & \text { on } \Gamma_{I}, & t \geq 0, \\
u_{F} \cdot \nu^{F}=u_{S} \cdot \nu^{F}, & \text { on } \Gamma_{I}, & t \geq 0, \\
\nabla p+\rho_{F} \frac{\partial^{2} u_{F}}{\partial t^{2}}=0, & \text { in } \Omega_{F}, & t>0, \\
p=-\rho_{F} c^{2} \operatorname{div} u_{F}, & \text { in } \Omega_{F}, & t \geq 0,
\end{array}
$$

plus initial conditions at $t=0$. In the previous equations $u_{S}$ and $u_{F}$ denote the solid and fluid displacements, respectively, $\rho_{S}$ and $\rho_{F}$ are the respective densities, $c$ the sound speed in the fluid, $p$ the acoustic pressure and $\sigma_{i j}$ the stress tensor in the solid, which is related to the strains by Hooke's constitutive law $\sigma_{i j}(v)=\lambda_{S} \sum_{k} \epsilon_{k k}(v) \delta_{i j}+2 \mu_{S} \epsilon_{i j}(v)$, where $\lambda_{S}$ and $\mu_{S}$ are the Lamé constants of the elastic structure and $\epsilon_{i j}(v):=\frac{1}{2}\left(\partial v_{i} / \partial x_{j}+\partial v_{j} / \partial x_{i}\right)$ is the linearized strain tensor.

In order to compute the natural vibration modes of the coupled system, we 
consider harmonic solutions for the displacements $u_{S}$ and $u_{F}$ and for the pressure $p$; that is, we seek solutions of equations (1-7) of the form

$$
\begin{aligned}
u_{S}(x, t)=u_{S}(x) e^{i \omega t}, & x \in \Omega_{S}, \quad t \geq 0, \\
u_{F}(x, t)=u_{F}(x) e^{i \omega t}, & x \in \Omega_{F}, \quad t \geq 0, \\
p(x, t)=p(x) e^{i \omega t}, & x \in \Omega_{F}, \quad t \geq 0,
\end{aligned}
$$

where $\omega>0$ is the angular frequency. We substitute these expressions in equations (1-7). In particular, observe that equation (6) reads

$$
\nabla p=\rho_{F} \omega^{2} u_{F}
$$

showing that the displacement field is conservative; i.e., there exist a displacement potential

$$
\varphi=\frac{p}{\rho_{F} \omega^{2}} \quad \text { such that } \quad u_{F}=\nabla \varphi, \quad \text { in } \Omega_{F} .
$$

All together, we obtain the following spectral problem:

Find $\lambda>0$ and functions $u, p$ and $\varphi$, not all identically zero, such that:

$$
\begin{aligned}
-\operatorname{div} \sigma(u)=\lambda \rho_{S} u, & \text { in } \Omega_{S}, \\
\sigma(u) \nu^{S}=0, & \text { on } \Gamma_{N}, \\
u=0, & \text { on } \Gamma_{D}, \\
-\sigma(u) \nu^{F}=\lambda \rho_{F} \varphi \nu^{F}, & \text { on } \Gamma_{I}, \\
\frac{\partial \varphi}{\partial \nu^{F}}=u \nu^{F}, & \text { on } \Gamma_{I}, \\
p=\lambda \rho_{F} \varphi, & \text { in } \Omega_{F}, \\
p=-\rho_{F} c^{2} \nabla^{2} \varphi, & \text { in } \Omega_{F},
\end{aligned}
$$

where $\lambda=\omega^{2}$ and $u_{S}$ has been replaced by $u$ for the sake of notational simplicity.

\section{$3 \quad$ Variational formulation in $(u, p, \varphi)$}

Standard methods to solve (8-14) consist of eliminating either the pressure or the displacement potential. However, in both cases non-symmetric eigenvalue problems are obtained (see for instance [11]). To avoid this drawback, Morand and Ohayon introduce in [8] an alternative procedure which consists of using both simultaneously. In this section we summarize their approach; further details and discussions can be found in their book [9].

In order to obtain a weak formulation for equations (8-14), firstly (8) is multiplied by a test function $v \in \mathcal{V}:=\left\{v \in H^{1}\left(\Omega_{S}\right)^{2} / v=0\right.$ on $\left.\Gamma_{D}\right\}$. Now, by 
integrating by parts in $\Omega_{S}$ (i.e., using Green's formula for tensor fields) and taking into account the boundary conditions (9-11), we obtain

$$
\int_{\Omega_{S}} \sigma(u): \epsilon(v) \mathrm{d} x-\lambda \int_{\Omega_{S}} \rho_{S} u \cdot v \mathrm{~d} x-\lambda \int_{\Gamma_{I}} \rho_{F} \varphi v \cdot \nu^{F} \mathrm{~d} s=0, \quad \forall v \in \mathcal{V},
$$

where $\sigma(u): \epsilon(v):=\sum_{i, j} \sigma_{i j}(u) \epsilon_{i j}(v)$. Secondly, multiplying (14) by a test function $\psi \in \mathcal{W}:=H^{1}\left(\Omega_{F}\right)$ and taking into account (12) we get

$$
-\int_{\Omega_{F}} \rho_{F} \nabla \varphi \cdot \nabla \psi \mathrm{d} x+\int_{\Gamma_{I}} \rho_{F} u \cdot \nu^{F} \psi \mathrm{d} s+\int_{\Omega_{F}} \frac{p \psi}{c^{2}} \mathrm{~d} x=0, \quad \forall \psi \in \mathcal{W} .
$$

Finally, multiplying (13) by an arbitrary test function $q \in \mathcal{Q}:=L^{2}\left(\Omega_{F}\right)$, we obtain:

$$
\int_{\Omega_{F}} \frac{p q}{\rho_{F} c^{2}} \mathrm{~d} x-\lambda \int_{\Omega_{F}} \frac{\varphi q}{c^{2}} \mathrm{~d} x=0, \quad \forall q \in \mathcal{Q} .
$$

Thus, we have the following (non-symmetric) variational formulation of problem (8-14):

Find $\lambda>0$ and $(u, p, \varphi) \in \mathcal{V} \times \mathcal{Q} \times \mathcal{W},(u, p, \varphi) \neq(0,0,0)$, such that equations (15-17) are satisfied.

On the other hand, the following alternative symmetric formulation in $(u, p, \varphi)$ is obtained by adding equations (15), (17) and $(-\lambda)$ times (16):

Find $\lambda>0$ and $(u, p, \varphi) \in \mathcal{V} \times \mathcal{Q} \times \mathcal{W},(u, p, \varphi) \neq(0,0,0)$, such that:

$$
\begin{gathered}
\int_{\Omega_{S}} \sigma(u): \epsilon(v) \mathrm{d} x+\int_{\Omega_{F}} \frac{p q}{\rho_{F} c^{2}} \mathrm{~d} x=\lambda\left[\int_{\Omega_{S}} \rho_{S} u \cdot v \mathrm{~d} x+m((u, p, \varphi),(v, q, \psi))\right], \\
\forall(v, q, \psi) \in \mathcal{V} \times \mathcal{Q} \times \mathcal{W},
\end{gathered}
$$

where the bilinear form $m(\cdot, \cdot)$ is defined by

$$
\begin{aligned}
& m((u, p, \varphi),(v, q, \psi)):=-\int_{\Omega_{F}} \rho_{F} \nabla \varphi \cdot \nabla \psi \mathrm{d} x \\
& +\left(\int_{\Gamma_{I}} \rho_{F} u \cdot \nu^{F} \psi \mathrm{d} s+\int_{\Gamma_{I}} \rho_{F} \varphi v \cdot \nu^{F} \mathrm{~d} s\right)+\left(\int_{\Omega_{F}} \frac{p \psi}{c^{2}} \mathrm{~d} x+\int_{\Omega_{F}} \frac{\varphi q}{c^{2}} \mathrm{~d} x\right) .
\end{aligned}
$$

It is simple to show that, for $\lambda>0$, the symmetric variational formulation (18-19) is equivalent to the non-symmetric one (15-17) in the sense that the corresponding eigenvalues and eigenfunctions are exactly the same. On the 
other hand, $\lambda=0$ is also an eigenvalue of both spectral problems but, as it is shown below, the corresponding eigenspaces differ.

The eigenspace asociated to $\lambda=0$ in equations $(15-17)$ is $\{(0,0, c), c \in \mathbf{R}\}$; i.e., null displacements and pressure and a constant potential. In fact, for $\lambda=0$, equations (15) and (17) implies $u=0$ and $p=0$, respectively, and then equation (16) yields

$$
\int_{\Omega_{F}} \rho_{F} \nabla \varphi \cdot \nabla \psi \mathrm{d} x=0, \quad \forall \psi \in \mathcal{W} ;
$$

in particular, by using $\psi=\varphi$, we conclude that $\varphi$ has to be constant in $\Omega_{F}$.

Instead, the variational formulation (18-19) has an infinite dimensional spurious eigenspace asociated to $\lambda=0$; this is the price that must be paid to have a symmetric formulation. In fact, equation (16) has been multiplied by $(-\lambda)$ and, therefore, for $\lambda=0$, this equation is not taken into account. Hence, as it can be easily checked, for any potential $\varphi \in \mathcal{W},(0,0, \varphi)$ is an eigenfunction of (18-19) corresponding to $\lambda=0$.

Now, the fact that for $\lambda=0$ the eigenspaces differ, does not affect the computation of the vibration modes of any fluid-solid system, since they always correspond to strictly positive eigenfrequencies. So, we are going to discretize the symmetric spectral problem (18-19) in order to obtain the corresponding matrix equations. Let us consider meshes in $\Omega_{F}$ and $\Omega_{S}$ with coinciding nodes at the interface. By using any finite element approximations of the variables we obtain:

$$
\left(\begin{array}{ccc}
K_{S} & 0 & 0 \\
0 & K_{P} & 0 \\
0 & 0 & 0
\end{array}\right)\left(\begin{array}{c}
U \\
P \\
\Phi
\end{array}\right)=\lambda\left(\begin{array}{ccc}
M & 0 & A \\
0 & 0 & B \\
A^{\mathrm{t}} & B^{\mathrm{t}} & -F
\end{array}\right)\left(\begin{array}{c}
U \\
P \\
\Phi
\end{array}\right)
$$

where $U, P$ and $\Phi$ are the vectors of nodal values for $u, p$ and $\varphi$, respectively, and the submatrices of (20) are defined by

$$
\begin{array}{ll}
\int_{\Omega_{S}} \sigma(u): \epsilon(v) \mathrm{d} x=V^{\mathrm{t}} K_{S} U, & \int_{\Omega_{F}} \frac{p q}{\rho_{F} c^{2}} \mathrm{~d} x=Q^{\mathrm{t}} K_{P} P, \\
\int_{\Omega_{S}} \rho_{S} u \cdot v \mathrm{~d} x=V^{\mathrm{t}} M U, & \int_{\Omega_{F}} \rho_{F} \nabla \varphi \cdot \nabla \psi \mathrm{d} x=\Psi^{\mathrm{t}} F \Phi, \\
\int_{\Gamma_{I}} \rho_{F} \varphi \nu^{F} \cdot v \mathrm{~d} s=V^{\mathrm{t}} A \Phi, & \int_{\Omega_{F}} \frac{\varphi q}{c^{2}} \mathrm{~d} x=Q^{\mathrm{t}} B \Phi,
\end{array}
$$

where $V, Q$ and $\Psi$ are the vectors of nodal values for $v, q$ and $\psi$, respectively. To solve this eigenvalue problem, Morand and Ohayon have proposed either 
the elimination of the potential nodal values $\Phi$ through the introduction of an added mass matrix ("mass coupling") or the elimination of the pressure nodal values $P$ through an added stiffnes matrix ("stiffness coupling"). The main disadvantage of these approachs is that both added matrices are not sparse.

No other attempts of solving this eigenproblem are known. Very likely this is because of the highly singular character of both matrices in (20): that in the left hand side has a huge kernel, and the one in the right hand side is indefinite.

We propose to solve directly (20). In the following section we show that, in spite of the fact that both matrices are singular, this eigenvalue problem is well posed. Moreover, we propose a particular finite element discretization for which we obtain an efficient and simple algorithm to compute these eigenvalues which involves dealing with symmetric sparse matrices and only one variable to describe the fluid.

\section{Efficient solution of the $(u, p, \varphi)$ formulation}

\subsection{Finite elements spaces}

In this formulation, the fluid is described redundantly by the pressure and the displacement potential. However, both variables do not need to be discretized in the same way. In fact, whereas the gradient of the potential is needed to compute the matrix $F$, no derivative of the pressure appears in this formulation. Therefore, we are allowed to use discontinuous piecewise constant elements to discretize the pressure.

Such choice has an aditional advantage: it allows a further elimination of the pressure preserving the sparse structure of the problem, as it is shown below. We describe the procedure in the $2 \mathrm{D}$ case, however the extension to $3 \mathrm{D}$ is straightforward. Let us consider triangular meshes in $\Omega_{S}$ and $\Omega_{F}$ with coinciding nodes at the interface. To compute the eigenmodes of the coupled system, we discretize the eigenvalue problem (20) by using:

- linear triangular elements for each component of the solid displacements,

- linear triangular elements for the displacement potential,

- piecewise constant elements for the pressure. 


\subsection{The algorithm}

For a coupled system, direct elimination of the pressure leads to a lack of symmetry. However, the pressure can be eliminated at a further stage. We describe our procedure for the most simple-minded method for computing a few eigenvalues: the inverse power method with shift. Similar ideas can be applied to more efficient "shift and invert" eigenvalue solvers like, for instance, Lanczos method.

Firstly, we prove that the intersection of the kernels of both matrices in (20) reduces to the null vector (this property is basic for any "shift and invert" eigenvalue solver to be well defined, as it is shown below in the case of the inverse power method). To prove it, let $\left(U_{*}, P_{*}, \Phi_{*}\right)$ be such that

$$
\left(\begin{array}{ccc}
K_{S} & 0 & 0 \\
0 & K_{P} & 0 \\
0 & 0 & 0
\end{array}\right)\left(\begin{array}{c}
U_{*} \\
P_{*} \\
\Phi_{*}
\end{array}\right)=\left(\begin{array}{l}
0 \\
0 \\
0
\end{array}\right) \quad \text { and } \quad\left(\begin{array}{ccc}
M & 0 & A \\
0 & 0 & B \\
A^{\mathrm{t}} & B^{\mathrm{t}} & -F
\end{array}\right)\left(\begin{array}{c}
U_{*} \\
P_{*} \\
\Phi_{*}
\end{array}\right)=\left(\begin{array}{l}
0 \\
0 \\
0
\end{array}\right)
$$

simultaneously. From the first homogeneous system of matrix equations, since $K_{S}$ and $K_{P}$ are non singular, we have that

$$
K_{S} U_{*}=0 \Longrightarrow U_{*}=0
$$

and

$$
K_{P} P_{*}=0 \Longrightarrow P_{*}=0,
$$

whereas, from the second system we know that

$$
B \Phi_{*}=0
$$

and

$$
A^{\mathrm{t}} U_{*}+B^{\mathrm{t}} P_{*}-F \Phi_{*}=0 .
$$

Now, replacing (21) and (22) into (24) we conclude that $F \Phi_{*}=0$, and taking into account the definition of $F$ it can be easily seen that this implies that $\Phi_{*}$ is constant. Finally, for constant vectors, by using the definition of $B$, it is simple to show that (23) is true only if $\Phi_{*}=0$.

The previous property shows that the eigenvalue calculation makes sense. For instance, given $\sigma>0$, if we look for the closest eigenvalue to $\sigma$ in (20), the shifted inverse power method reads: 
Input: $U^{0}, P^{0}$ and $\Phi^{0}$.

For $m:=0,1,2, \ldots$, until a stop criterion is attained, solve:

$$
\left[\left(\begin{array}{ccc}
K_{S} & 0 & 0 \\
0 & K_{P} & 0 \\
0 & 0 & 0
\end{array}\right)-\sigma\left(\begin{array}{ccc}
M & 0 & A \\
0 & 0 & B \\
A^{\mathrm{t}} & B^{\mathrm{t}} & -F
\end{array}\right)\right]\left(\begin{array}{l}
U^{m+1} \\
P^{m+1} \\
\Phi^{m+1}
\end{array}\right)=\left(\begin{array}{ccc}
M & 0 & A \\
0 & 0 & B \\
A^{\mathrm{t}} & B^{\mathrm{t}} & -F
\end{array}\right)\left(\begin{array}{c}
U^{m} \\
P^{m} \\
\Phi^{m}
\end{array}\right) .
$$

Notice that, since the intersection of the kernels of both matrices in (20) reduces to the null vector, then the matrix in the left hand side of this algorithm is non singular, except if $\sigma$ is already one of the eigenvalues we are looking for.

At this instance, we can eliminate $P$. In fact, the second matrix equation can be written

$$
P^{m+1}=K_{P}^{-1}\left[\sigma B \Phi^{m+1}+B \Phi^{m}\right]
$$

and from the third matrix equation we get

$$
-\sigma A^{\mathrm{t}} U^{m+1}-\sigma B^{\mathrm{t}} P^{m+1}+\sigma F \Phi^{m+1}=A^{\mathrm{t}} U^{m}+B^{\mathrm{t}} P^{m}-F \Phi^{m} .
$$

Now, replacing (25) into (26), we obtain

$$
-\sigma A^{\mathrm{t}} U^{m+1}+\sigma[F-\sigma C] \Phi^{m+1}=A^{\mathrm{t}} U^{m}+[2 \sigma C-F] \Phi^{m}+C \Phi^{m-1},
$$

where $C:=B^{\mathrm{t}} K_{P}^{-1} B$. Notice that $C$ is sparse because $K_{P}$ is a diagonal matrix and $B$ is sparse. Furthermore, the entries of $C$ can be explicitely written

$$
C_{i j}=\frac{\rho_{F}}{9 c^{2}} \sum_{T \ni P_{i}, P_{j}}|T|
$$

where $P_{i}$ and $P_{j}$ denotes the vertices numbered $i$ and $j$ in the fluid mesh, respectively, and $|T|$ stands for the triangle area.

Thus, combining (27) with the first matrix equation in the algorithm we get

$$
\begin{aligned}
& \left(\begin{array}{cc}
K_{S}-\sigma M & -\sigma A \\
-\sigma A^{\mathrm{t}} & \sigma(F-\sigma C)
\end{array}\right)\left(\begin{array}{l}
U^{m+1} \\
\Phi^{m+1}
\end{array}\right) \\
& \quad=\left(\begin{array}{cc}
M & A \\
A^{\mathrm{t}} & 2 \sigma C-F
\end{array}\right)\left(\begin{array}{l}
U^{m} \\
\Phi^{m}
\end{array}\right)+\left(\begin{array}{c}
0 \\
C \Phi^{m-1}
\end{array}\right) .
\end{aligned}
$$

Therefore, denoting $\Psi^{m}:=\sigma \Phi^{m}+\Phi^{m-1}$, we obtain the following algorithm equivalent to the previous one, but not involving $P$ : 
Input: $U^{0}, \Phi^{0}$ and $\Phi^{-1}$.

For $m:=0,1,2, \ldots$, until a stop criterion is attained,

let $\Psi^{m}:=\sigma \Phi^{m}+\Phi^{m-1}$

and solve:

$$
\begin{array}{r}
{\left[\left(\begin{array}{cc}
K_{S} & 0 \\
0 & 0
\end{array}\right)-\sigma\left(\begin{array}{cc}
M & A \\
A^{\mathrm{t}} & \sigma C-F
\end{array}\right)\right]\left(\begin{array}{c}
U^{m+1} \\
\Phi^{m+1}
\end{array}\right)} \\
\quad=\left(\begin{array}{cc}
M & A \\
A^{\mathrm{t}} & \sigma C-F
\end{array}\right)\left(\begin{array}{c}
U^{m} \\
\Phi^{m}
\end{array}\right)+\left(\begin{array}{c}
0 \\
C \Psi^{m}
\end{array}\right)
\end{array}
$$

The initial value of $\Phi^{-1}$ can be simply set equal to zero.

Finally, the pressure can be recovered either by computing

$$
P^{m+1}=K_{P}^{-1} B \Psi^{m+1}
$$

or by means of equation (13) in terms of the potential. Let us remark that all the matrices in the given algorithm are symmetric and sparse and they involve only one degree of freedom per vertex in the fluid.

Also notice that the proposed algorithm is theoretically equivalent to apply classical shifted inverse iteration method to the original eigenproblem (20). Thus, all the properties of the latter remain valid in our algorithm. For instance, Rayleigh quotients can be computed by using (29) to compute the eliminated pressure variables at each step, and these quotients could be used to change shifts in order to attempt improving the convergence of the eigensolver.

\subsection{Theoretical and computational validation in a rigid cavity}

As a first validation for our proposed algorithm we compute the acoustic modes of a fluid enclosed by a rectangular rigid cavity. In this case there exists an analytical expression for the eigenfrequencies. Let $A$ and $B$ be the lenghts of the edges of the rectangle which is supossed to be completely filled by the fluid. Then, the exact eigenfrequencies (in $\mathrm{rad} / \mathrm{s}$ ) are given by

$$
\omega_{n m}=\sqrt{\lambda_{n m}}=c \pi \sqrt{\left(\frac{n}{A}\right)^{2}+\left(\frac{m}{B}\right)^{2}}, \quad n, m=0,1,2, \ldots(n+m \neq 0) .
$$

In Table 1 we present the lowest eigenfrequencies (in rad/s) computed by our algorithm with several embedded uniform meshes with increasing number of degrees of freedom (d.o.f.). We consider a rectangular rigid cavity with air inside: $A=1 \mathrm{~m}, B=0.8 \mathrm{~m}$ and $c=340 \mathrm{~m} / \mathrm{s}$. We also include in the table the corresponding exact eigenfrequencies. 
Table 1

Air in a rigid cavity: computed and exact eigenfrequencies.

\begin{tabular}{cccccc}
\hline Mode & d.o.f. $=81$ & d.o.f. $=289$ & d.o.f. $=625$ & d.o.f. $=1089$ & Exact \\
\hline$\omega_{10}$ & 1079.5330 & 1070.9940 & 1069.4110 & 1068.8560 & 1068.142 \\
$\omega_{01}$ & 1349.3060 & 1338.7330 & 1336.7610 & 1336.0690 & 1335.177 \\
$\omega_{11}$ & 1750.9890 & 1720.2270 & 1714.4790 & 1712.4610 & 1709.861 \\
$\omega_{20}$ & 2228.5890 & 2159.1500 & 2146.4420 & 2141.9980 & 2136.283 \\
$\omega_{21}$ & 2685.6980 & 2560.3750 & 2537.4950 & 2529.4950 & 2519.206 \\
$\omega_{02}$ & 2785.8490 & 2698.8740 & 2683.0340 & 2677.4910 & 2670.353 \\
$\omega_{12}$ & 3052.1140 & 2920.6330 & 2895.9640 & 2887.2790 & 2876.059 \\
$\omega_{30}$ & 3546.8950 & 3283.4130 & 3239.0720 & 3223.8290 & 3204.424 \\
\hline
\end{tabular}

An excelent performance of the method can be clearly observed. Furthermore, since the exact eigenfrequencies are known, the order of convergence can be easily computed; by so doing, the following estimate is obtained

$$
\left|\omega_{n m}^{h}-\omega_{n m}\right| \leq C h^{2}
$$

where $h$ denotes the stepsize of the mesh and $\omega_{n m}^{h}$ the corresponding computed eigenfrequency.

Such good convergence could be theoretically anticipated: for a rigid cavity this method turns out to be equivalent to a piecewise linear discretization of the pure potential formulation:

$$
\int_{\Omega_{F}} \rho_{F} \nabla \varphi \cdot \nabla \psi \mathrm{d} x=\lambda \int_{\Omega_{F}} \frac{\rho_{F}}{c^{2}} \varphi \psi \mathrm{d} x, \quad \forall \psi \in \mathcal{W} .
$$

In fact, for the rigid cavity problem, $u=0$ and so from (20) we obtain

$$
\left(\begin{array}{cc}
K_{P} & 0 \\
0 & 0
\end{array}\right)\left(\begin{array}{c}
P \\
\Phi
\end{array}\right)=\lambda\left(\begin{array}{cc}
0 & B \\
B^{\mathrm{t}} & -F
\end{array}\right)\left(\begin{array}{c}
P \\
\Phi
\end{array}\right)
$$

Now, in this case, the pressure could be effectively eliminated yielding

$$
F \Phi=\lambda C \Phi
$$

with $C=\left(B^{\mathrm{t}} K_{P}^{-1} B\right)$ being the matrix given in (28). Let us remark that the entries of this matrix $C$ are exactly the same that are obtained if the integral in the right hand side of (30) is computed by means of the midppoint integration rule. On the other hand $F$ is exactly the matrix of the left hand side of the same equation. Hence, for the rigid cavity problem, our algorithm coincides 
with a simple consistent discretization of the standard potential formulation (30) and, consequently, produce the same results.

\subsection{Computation of elastoacoustic modes}

Now, to show the efectiveness of our approach in a coupled problem, we present a numerical example that consists of a square closed steel vessel completely filled with water as shown in Figure 2.

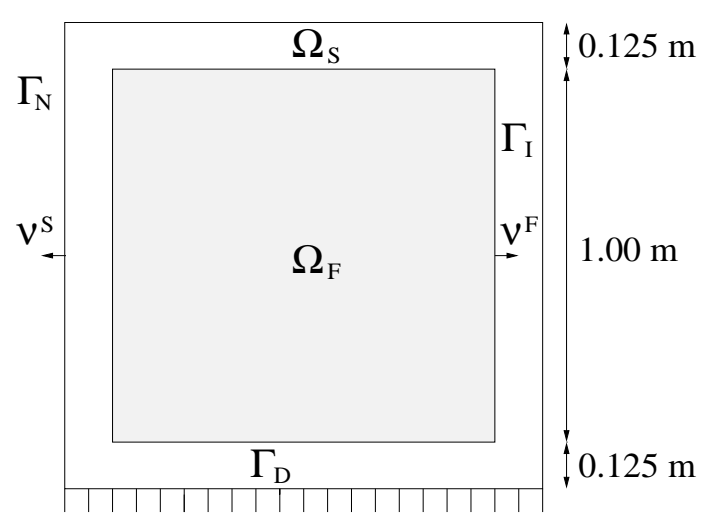

Fig. 2. Steel cavity with fluid inside.

For the fluid we have used the physical parameters:

- $\rho_{F}=1000 \mathrm{Kg} / \mathrm{m}^{3}$,

- $c=1430 \mathrm{~m} / \mathrm{s}$,

and for the steel we have taken:

- $\rho_{S}=7700 \mathrm{Kg} / \mathrm{m}^{3}$,

- Young Modulus = $1.44 \times 10^{11} \mathrm{~Pa}$,

- Poisson ratio $=0.35$.

In Table 2 we present the lowest eigenfrequencies (in $\mathrm{rad} / \mathrm{s}$ ) calculated with this method for three succesively refined meshes. In order to validate these results, we include in the last column the eigenfrequencies computed on the most refined mesh by means of a pure displacement formulation described in $[2]$, which has been proved in $[1,10]$ to converge to the exact solution with optimal order.

The very good convergence of our method can be observed from the first three columns of the table. On the other hand, the two last columns corresponding to the eigenfrequencies computed by both methods on a same mesh show an excellent agreement. However let us remark that, even using the same mesh, 
the number of degrees of freedom is significatively larger for the method in [2], since it is based on discretizing the fluid displacement vector field.

Table 2

Air in a steel cavity: computed eigenfrequencies.

\begin{tabular}{ccccc}
\hline & \multicolumn{3}{c}{ Our algorithm } & Method in [2] \\
\cline { 2 - 4 } Mode & d.o.f.= 721 & d.o.f.=1489 & d.o.f.=2529 & d.o.f.=4576 \\
\hline$\omega_{1}$ & 552.480 & 478.496 & 446.373 & 446.354 \\
$\omega_{2}$ & 1884.787 & 1661.850 & 1562.892 & 1562.601 \\
$\omega_{3}$ & 3019.979 & 2694.687 & 2541.073 & 2540.501 \\
$\omega_{4}$ & 3766.833 & 3547.817 & 3434.404 & 3433.510 \\
$\omega_{5}$ & 4401.758 & 4260.563 & 4181.036 & 4178.811 \\
$\omega_{6}$ & 4805.353 & 4612.528 & 4520.354 & 4516.986 \\
$\omega_{7}$ & 5526.067 & 5408.118 & 5343.842 & 5340.046 \\
$\omega_{8}$ & 5834.725 & 5710.485 & 5664.825 & 5662.969 \\
$\omega_{9}$ & 6436.497 & 6182.945 & 6009.386 & 6004.506 \\
\hline
\end{tabular}

\subsection{A problem with a free interface}

The proposed methodology works adequately well also for fluids with a free surface condition. We present a numerical example that consists of an open rectangular vessel of steel containing water as shown in Figure 3; the fluid has a free surface at constant atmospheric pressure. We neglect surface tension and gravity effects, therefore no low frequency sloshing modes will be present.

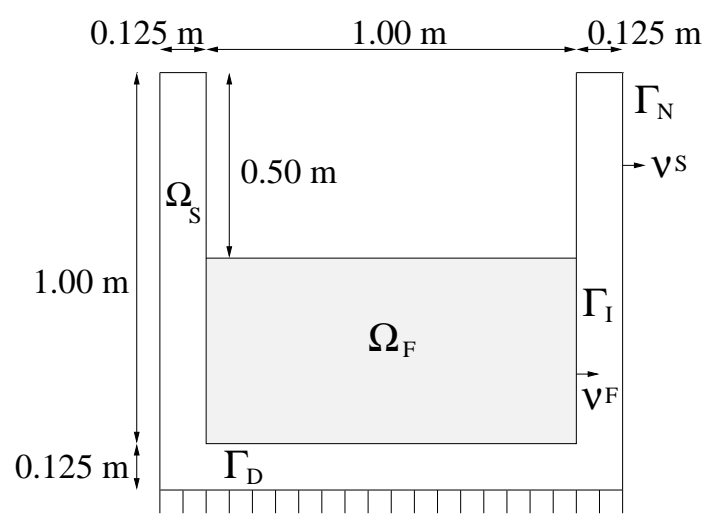

Fig. 3. Liquid in an open vessel. 
We consider $p=0$ in the free surface and hence, by virtue of $(13), \varphi=0$. So, to adequate this method to a free surface condition it is simply necessary setting the potential values to zero at the vertices belonging to the free surface.

We have used the same values of the physical parameters as in the previous example.

In Table 3 we show the lowest eigenfrequencies (in $\mathrm{rad} / \mathrm{s}$ ) of this coupled system computed with different meshes. In order to compare, we include again in the last column the results obtained with the method in [2] using the most refined mesh. Once more the good performance of our method and an excellent agreement with that in [2] can be clearly observed. Notice that the method in [2] requires again a larger number of degrees of freedom for the same mesh.

Table 3

Water in an open steel vessel: computed eigenfrequencies.

\begin{tabular}{ccccc}
\hline & \multicolumn{3}{c}{ Our algorithm } & Method in [2] \\
\cline { 2 - 4 } Mode & d.o.f.=471 & d.o.f.=957 & d.o.f.=1611 & d.o.f.= 2634 \\
\hline$\omega_{1}$ & 728.479 & 624.475 & 581.111 & 580.966 \\
$\omega_{2}$ & 728.775 & 624.727 & 581.344 & 581.202 \\
$\omega_{3}$ & 3763.547 & 3367.454 & 3175.117 & 3171.749 \\
$\omega_{4}$ & 3928.191 & 3448.708 & 3236.361 & 3232.958 \\
$\omega_{5}$ & 4777.027 & 4641.106 & 4599.095 & 4595.590 \\
$\omega_{6}$ & 6439.768 & 6298.712 & 6221.650 & 6213.073 \\
$\omega_{7}$ & 6674.476 & 6615.959 & 6589.391 & 6589.274 \\
$\omega_{8}$ & 6674.617 & 6621.981 & 6601.989 & 6601.636 \\
\hline
\end{tabular}

\section{Conclusions}

Morand and Ohayon's formulation to compute elastoacoustic vibrations has been revisited. In the fluid we consider a discretization by discontinuous constant elements for the pressure and linear triangular elements for the displacement potential. Such discretization allows a further elimination of pressure degrees of freedom without loosing neither the symmetry nor the sparseness of the involved matrices. An algorithm for the shifted power method is given to compute the vibration modes; the same idea works for any "shift and invert" method.

When applied to compute pure acoustic modes this discretization coincides with that of a standard potential formulation of the problem. For coupled 
problems, with or without free surfaces, the obtained numerical results almost coincide with those obtained with a pure displacement formulations on the same mesh, but the latter requires much more degrees of freedom for the fluid. This displacement formulation has been proved to converge with optimal order. During the revision of the present paper a similar result has been proved for the method we consider herein (see [3]).

\section{References}

[1] A. Bermúdez, R. Durán, M.A. Muschietti, R. Rodríguez and J. Solomin, Finite element vibration analysis of fluid-solid systems without spurious modes, SIAM J. Numer. Anal. 32 (1995) 1280-1295.

[2] A. Bermúdez and R. Rodríguez, Finite element computation of the vibration modes of a fluid-solid system, Comput. Methods Appl. Mech. and Engrg. 119 (1994) 355-370.

[3] A. Bermúdez, R. Rodríguez, Analysis of a finite element method for pressure/potential formulation of elastoacoustic spectral problems. Technical Report DIM 99-05, Universidad de Concepción, Concepción, 1999.

[4] H.C. Chen and R.L. Taylor, Vibration analysis of fluid-solid systems using a finite element displacements formulation, Internat. J. Numer. Methods Engrg. 29 (1990) 683-698.

[5] C. Conca, J. Planchard, M. Vanninathan, Fluids and Periodic Structures (Masson, Paris, 1992).

[6] G.C. Everstine, A symmetric potential formulation for fluid-structure interaction, J. Sound Vib. 79 (1981) 157-160.

[7] L. Kiefling and G.C. Feng, Fluid-structure finite element vibration analysis, AIAA J. 14 (1976) 199-203.

[8] H.J-P. Morand and R. Ohayon, Substructure variational analysis of the vibrations of coupled fluid-structure systems. Finite element results, Internat. J. Numer. Methods Engrg. 14 (1979) 741-755.

[9] H.J-P. Morand and R. Ohayon, Fluid Structure Interaction (John Wiley \& Sons, 1995).

[10] R. Rodríguez and J. Solomin, The order of convergence of eigenfrequencies in finite element approximations of fluid-structure interaction problems, Math. Comp. 65 (1996) 1463-1475.

[11] O.C. Zienkiewicz and R.L.Taylor, The Finite Element Method, (McGraw-Hill, Vol. 2, 1989). 